\title{
48
}

\section{CORONAVIRUS ENVELOPE ASSEMBLY IS SENSITIVE TO CHANGES IN THE TERMINAL REGIONS OF THE VIRAL M PROTEIN}

\author{
C. A. M. de Haan, H. Vennema, and P. J. M. Rottier \\ Institute of Virology \\ Department of Infectious Diseases and Immunology \\ Faculty of Veterinary Medicine, and Institute of Biomembranes \\ Utrecht University, Yalelaan 1 \\ 3584 CL Utrecht, The Netherlands
}

\section{ABSTRACT}

Recently we demonstrated that the co-expressed coronavirus membrane proteins have the capacity to assemble viral envelopes which are similar to normal virus particles in dimensions and appearance, and which can form independent of a nucleocapsid (Vennema et al., 1996). For the formation of these particles only the $M$ and the $E$ protein are required; the $\mathrm{S}$ protein is dispensable but is incorporated when present. As we illustrate here, this virus-like particle assembly system is an ideal tool to study the interactions between the essential assembly partners $\mathrm{M}$ and $\mathrm{E}$ in molecular detail. Taking a mutagenetic approach we demonstrate that envelope assembly is critically sensitive to changes in the primary structure of both terminal domains of the $M$ protein. The effects were most dramatically observed after mutation of the carboxy-terminal domain where the deletion of just one single amino acid at the extreme terminus abolished particle formation almost completely. But also some subtle mutations in the amino-terminal domain were severely inhibitory to the assembly process. Interestingly, mutant $\mathrm{M}$ proteins that were themselves incompetent to support particle formation appeared to inhibit, in a concentration dependent manner, the assembly of particles by wild-type $\mathrm{M}$ and $\mathrm{E}$ protein.

\section{INTRODUCTION}

Enveloped viruses assemble by budding through cellular membranes. The viral nucleocapsid or core, present in the cytoplasm, meets the viral envelope proteins at a specified cellular membrane and their interactions lead to the formation of a virion. Often, as is 
the case for togaviruses (Suomalainen et al., 1992; Hobman et al., 1994), these interactions are an absolute requirement for budding to occur. For several viruses, however, the envelope proteins are not required: retroviruses (Delchambre et al., 1989; Gheysen et al., 1989) and rhabdoviruses (Mebatsion et al., 1996) allow budding of their nucleocapsids without the involvement of these proteins, giving rise to noninfectious virions. Conversely, a number of other viruses have been found to assemble "empty" particles just from their envelope proteins, i.e. without the need for a nucleocapsid. Usually these capsidless viral particles are smaller than the normal virions, as observed with flaviviruses (Schalich et al., 1996), and morphologically different, as seen with hepatitis B virus (Simon et al., 1988). This is, however, not the case for coronaviruses. The membrane proteins of these viruses can drive the formation of envelopes which, when viewed by electron microscopy, look perfectly similar to authentic virions (Vennema et al., 1996).

Of the four coronaviral envelope proteins only two are actually essential for budding: the membrane $(M)$ protein and the envelope $(E)$ protein. Coronavirions without the spike (S) protein, and thus without spikes, have been demonstrated already a long time ago (e.g. Rottier et al., 1981). The haemagglutinin-esterase (HE) protein occurs only in a subset of coronaviruses. The triple-spanning $\mathrm{M}$ protein is the major component of the viral envelope. In mouse hepatitis virus (MHV) it has its carboxy-terminus inside the particle while the amino-terminus, which carries an oligosaccharide side chain linked to threonine, protrudes from the outside of the envelope (see Rottier, 1995). Of the E protein the carboxy-terminus is also facing the virion inside but little if anything of this protein seems to be exposed on the outside (M. Raamsman, H. Vennema and P. Rottier, unpublished data).

Coexpression of $\mathrm{M}$ and $\mathrm{E}$ in cells gives rise to the synthesis of the virus-like particles (VLPs) mentioned above. This provides us with a very simple assay system to investigate the primary structure requirements of these two proteins for envelope formation. In this study we have focussed on the terminal parts of the M protein.

\section{MATERIALS AND METHODS}

\subsection{Expression Vectors and Site-Directed Mutagenesis}

Expression construct pTM5ab contains the MHV mRNA 5 open reading frames (ORFs) 5a and 5b, the latter encoding the E protein, in pTUG3 (Vennema et al., 1996). Expression construct pTUMM contains the MHV M gene cloned as a Xhol fragment into pTUG3. All mutant $M$ proteins were generated by standard polymerase chain reaction (PCR) cloning methods and cloned into pTUG3, except mutants $\Delta \mathrm{N}, \mathrm{G}_{11} \mathrm{~N}_{13}$ and $\Delta \mathrm{C}$. These were made as described previously (Rottier et al., 1990), and expressed from the transcription vector pTZ19R (Mead et al., 1986).

\subsection{Expression, Metabolic Labeling, and Immunoprecipitation}

Subconfluent monolayers of OST-7 cells were infected with recombinant vaccinia virus encoding the T7 RNA polymerase (vTF7-3) and subsequently transfected with expression constructs as described before (Vennema et al., 1996). Metabolic labeling of the transfected cells with ${ }^{35} \mathrm{~S}$ methionine was performed essentially as described by de Vries et al. (1995) for three hours starting at $\mathrm{t}=5$. Rabbit anti-MHV serum \#134 (Rottier et al., 1981) was used at a 500-fold dilution for immunoprecipitation of MHV proteins from cell lysates and cell culture media as described before (de Vries et al., 1995). 


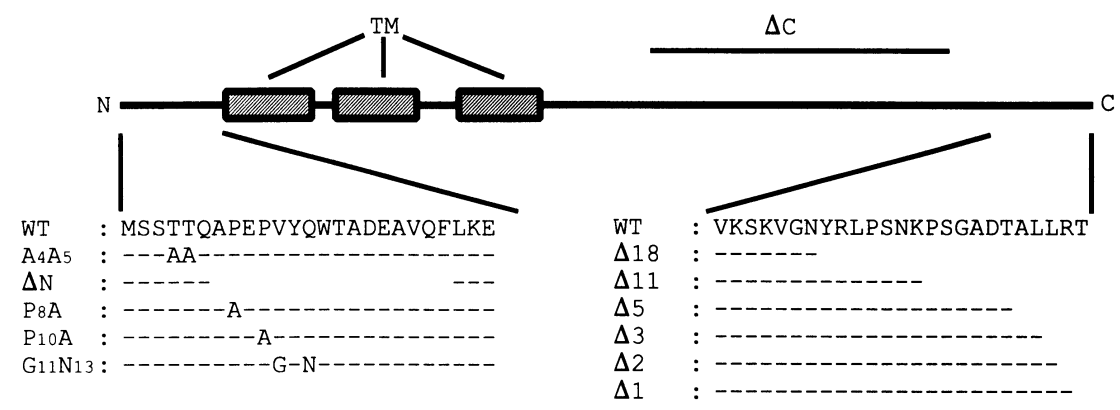

Figure 1. Overview of mutant $M$ proteins. On top a schematic representation of the structure of the $M$ protein. The three transmembrane (TM) domains are indicated by boxes. The left and right panel show amino acid sequences of the amino-terminal and carboxy-terminal domain, respectively, and the mutations in these domains. Dashes represent unchanged residues, a gap refers to a deletion. The domain deleted in mutant $\Delta \mathrm{C}$ (residues $\mathrm{E}_{121}$ through $D_{195}$ ) is indicated by a horizontal line.

\section{RESULTS}

\subsection{The MHV M Cytoplasmic Domain in VLP Assembly}

In this study we analyzed the primary structure requirements of the $M$ protein for MHV envelope assembly. To this aim we constructed a number of M mutants.

Our first set of mutants (Fig. 1) consisted of a panel of molecules with progressively smaller deletions at the carboxy-terminus, ranging from 18 till 1 amino acids (aa), as well as one internal deletion mutant $(\Delta C)$, from which $E_{121}$ through $D_{195}$, which comprise most of the amphiphilic domain, were removed. The ability of these mutant $\mathrm{M}$ molecules to function in assembly was tested by coexpression of each of the mutant genes together with the E protein gene. Genes were expressed using the recombinant vaccinia virus/bacteriophage T7 RNA polymerase system in OST-7 cells and labeled with ${ }^{35} \mathrm{~S}$ methionine/cysteine from 5 to $8 \mathrm{~h}$ postinfection. Cells and media were collected separately and viral proteins were immunoprecipitated with MHV-specific antibodies followed by SDS-PAGE in a $15 \%$ gel (Fig. 2). The analysis of the cell lysates of the single expressions demonstrates that all mutant constructs were expressed, yielding products of expected sizes. In all cases the $M$ proteins appear as a set of proteins differing in apparent molecular weight, due to different extents of O-glycosylation. The patterns of the glycosylated species of $\mathrm{M}$ mutants are not much different from that of the wild-type (WT), indicating that the mutations in the C-terminus did not affect the ability of the N-terminus to become glycosylated, nor did they affect the ability of the proteins to be transported to the Golgi complex. In the double expressions, the presence of the E protein did not seem to affect the synthesis of $\mathrm{M}$ quantitatively or qualitatively under the experimental conditions used. The E protein is not resolved in a $15 \%$ gel, but was synthesized as was shown using an E-specific serum (not shown). Particle assembly and secretion was assayed by measuring the release of the $\mathrm{M}$ protein into the culture medium. The E protein is extremely difficult to detect in VLPs, due to its small size and very low abundance. When looking at WT-M it is clear that $\mathrm{M}$ release into the medium is critically dependent on the presence of the $\mathrm{E}$ protein, consistent with our earlier findings (Vennema et al., 1996). However, all mutant M pro- 


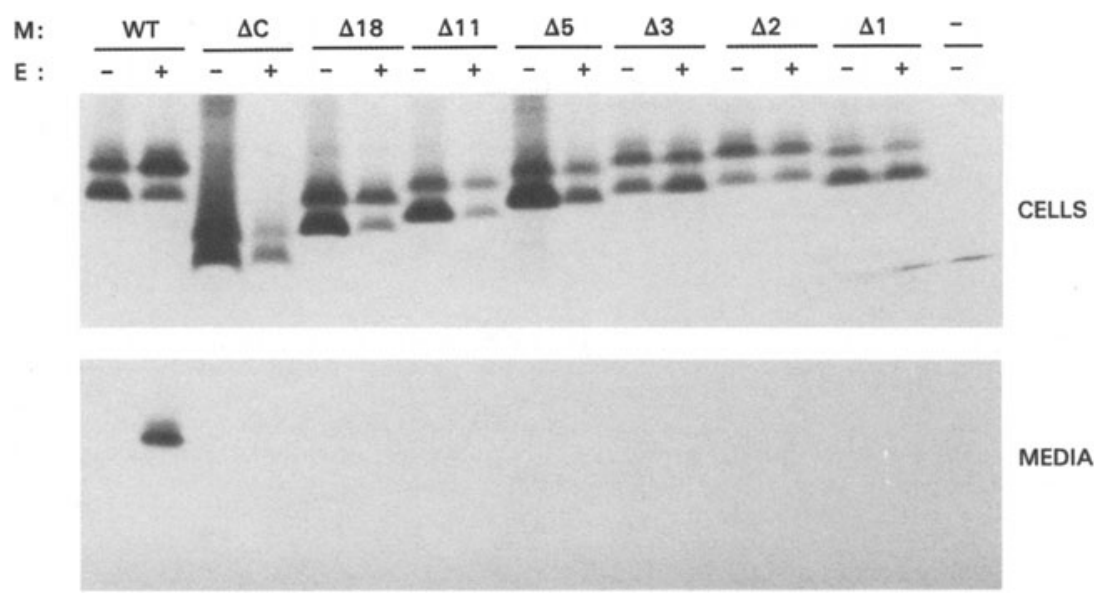

Figure 2. Effect of deletions in the M cytoplasmic domain on VLP assembly. Recombinant vaccinia virus vTF7-3 infected OST7-1 cells were transfected with a plasmid containing the wild-type (WT) or mutant M gene either alone or in combination with a plasmid containing the $\mathrm{E}$ protein gene, each gene behind a $\mathrm{T} 7$ promoter. Cells were labeled for $3 \mathrm{~h}$ with ${ }^{35} \mathrm{~S}$-labeled amino acids. Both cells (upper panel) and the culture medium (lower panel) were prepared and used for immunoprecipitation and the precipitates were analyzed by SDS-PAGE. The different M genes expressed are indicated above each set.

teins fail to be secreted into the culture medium when expressed in combination with the $\mathrm{E}$ protein. Not only an internal deletion in the cytoplasmic domain but also deletions at the C-terminus are fatal for VLP assembly. Even the deletion of only one single amino acid at the extreme C-terminus abolished VLP formation almost completely (Fig. 2).

\subsection{The MHV M Amino-Terminal Domain in VLP Assembly}

In order to investigate the role of the amino-terminal domain of M in VLP formation, a second set of mutants was constructed (Fig. 1), consisting of a panel of molecules with several mutations in this domain. In mutant $\mathrm{A}_{4} \mathrm{~A}_{5}$ threonines at position 4 and 5 are substituted by alanines. Mutant $\Delta \mathrm{N}$ lacks $\mathrm{A}_{7}$ through $\mathrm{F}_{22}$, an internal deletion of 16 aa. Three mutants have substitutions within this deleted sequence. In mutant $\mathrm{P}_{8} \mathrm{~A}$ the proline residue at position 8 is substituted by alanine; $\mathrm{P}_{10} \mathrm{~A}$ has a similar substitution of the proline at position 10. Mutant $\mathrm{G}_{11} \mathrm{~N}_{13}$ has substitutions of valine and glutamine at positions 11 and 13 into glycine and asparagine, respectively. The mutant constructs were expressed alone and in combination with the E protein gene. Cells and media were processed and analyzed as in the previous experiment. The analysis of the cell lysates (Fig. 3) shows, that all mutants were expressed to yield products of expected sizes. Mutant $G_{11} N_{13}$ shows the same glycosylation pattern as WT-M indicating that this mutant is transported at least to the Golgi complex. Mutants $\mathrm{P}_{8} \mathrm{~A}$ and $\mathrm{P}_{10} \mathrm{~A}$ also become glycosylated but to a lesser extent. This is, however, not a reflection of the inability of the mutants to be transported to the Golgi apparatus, as was verified by immunofluorescence (not shown), but substitution of the prolines results in less efficient glycosylation at the $\mathrm{N}$-terminal end. Mutants $\mathrm{A}_{4} \mathrm{~A}_{5}$ and $\Delta \mathrm{N}$ do not become glycosylated at all and, again, this is not a reflection of their inability 


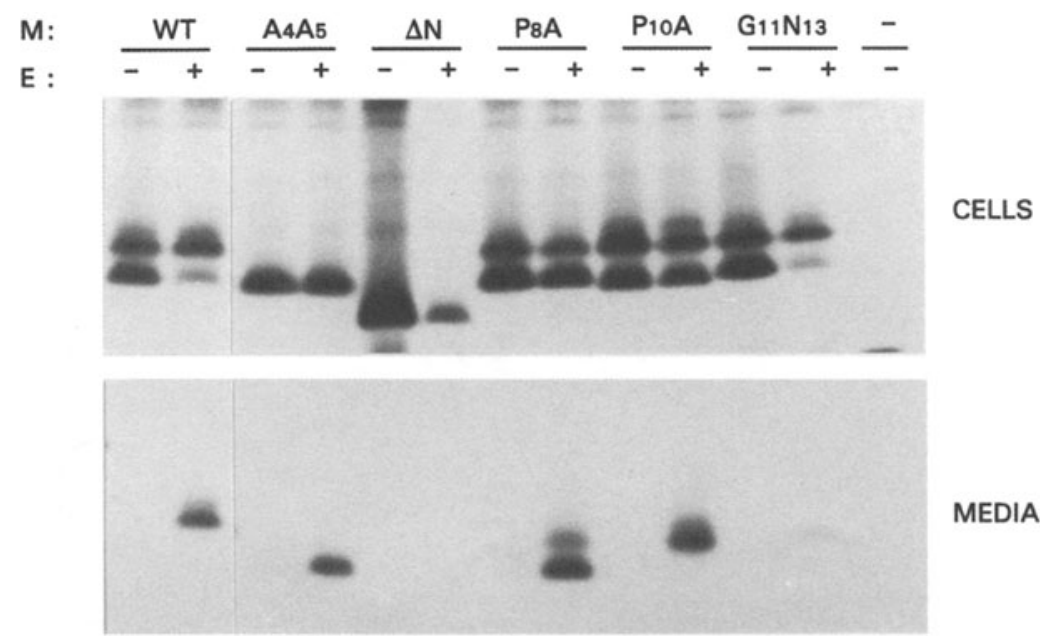

Figure 3. Effect of mutations in the amino-terminal domain of $M$ on VLP assembly. $M$ and $E$ genes were expressed as described in the legend to Fig. 2.

to be transported to the Golgi complex, but results from their mutations being fatal for glycosylation at the N-terminus. The presence of the $\mathrm{E}$ protein does not seem to affect the synthesis of mutant $\mathrm{M}$ quantitatively or qualitatively under the experimental conditions used. All mutant $M$ proteins fail to be secreted into the medium in the single expressions. When coexpressed with the E protein, mutant $\mathrm{A}_{4} \mathrm{~A}_{5}$ is secreted into the medium with the same efficiency as WT-M. This result indicates that the threonines are not a primary structure requirement for VLP formation. Furthermore, O-glycosylation of the M protein is also not a prerequisite for VLP assembly and release. Mutant $\Delta \mathrm{N}$ is not secreted into the medium at all; the 16 aa deletion for some reason abolishes the $M$ protein's ability to be assembled into VLPs. Since $\mathrm{P}_{8} \mathrm{~A}$ and $\mathrm{P}_{10} \mathrm{~A}$ are secreted efficiently the prolines do not seem important for VLP assembly. In contrast, secretion of $G_{11} N_{13}$ is decreased significantly, indicating that envelope-assembly is sensitive to changes in the region downstream of the prolines (Fig. 3).

\subsection{Inhibition of VLP Formation by Assembly-Incompetent Mutant M Proteins}

Our next aim was to investigate whether mutants of $M$ which are themselves incompetent in VLP formation have an effect on assembly of VLPs by WT-M and E. Therefor, a triple expression experiment was performed, expressing WT-M, E and mutant M. Fixed amounts of plasmid DNA coding WT-M and E were used in the transfections, while of the plasmid DNA coding for mutant $\mathrm{M}$ an amount equal to or smaller than that of WT-M was used. Cells and media were processed and analyzed similarly as in the previous experiments. The analysis of the cell lysates shows the differently glycosylated $M$ species (Fig. 4). Mutant $\Delta 1$ can not be distinguished from WT-M, due to their small size difference. Of mutant $\Delta 18$ the unglycosylated form can clearly be discriminated from WT-M; 


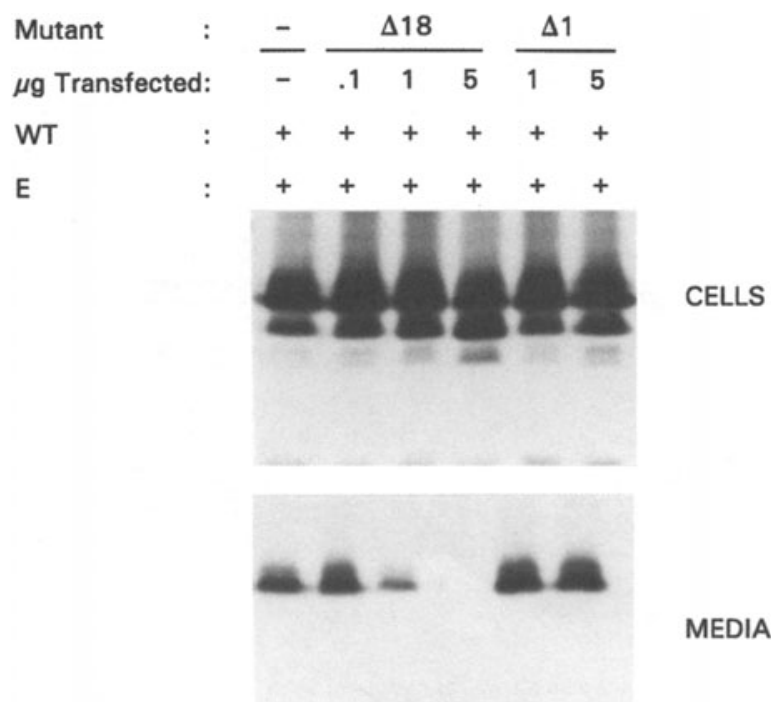

Figure 4. Inhibition of VLP formation by carboxy-terminal deletion mutant $M$ proteins. After infection of OST7-1 cells with recombinant vaccinia virus vTF7-3, cells were transfected with plasmid DNA encoding WT M and $\mathrm{E}(5 \mu \mathrm{g}$ each) together with $0.1,1$ or $5 \mu \mathrm{g}$ plasmid DNA encoding mutant $\mathrm{M}$. The different $\mathrm{M}$ mutants expressed are indicated above each set. Cells were labeled for $3 \mathrm{~h}$ with ${ }^{35} \mathrm{~S}$-labeled amino acids. Both cells (upper panel) and the culture medium (lower panel) were prepared for immunoprecipitation and the precipitates were analyzed by SDS-PAGE.

the glycosylated $\Delta 18$ species, however, comigrates with the unglycosylated form of WTM. The glycosylated species of WT-M run slower and can thus be discriminated from the $\Delta 18$ proteins. When equal amounts of plasmid DNA of WT-M and $\Delta 18$ were co-transfected, products from both were clearly detected in the cell lysate, although the expression level of the mutant seems much lower than that of WT. Using lower amounts of mutant $\Delta 18$ plasmid DNA led to lower amounts of the unglycosylated $\Delta 18$ species that were immunoprecipitated, while the amounts of glycosylated WT-M forms was not noticeably changed. Analysis of the culture media showed that that the amount of WT-M secreted was reduced when $\Delta 18$ was coexpressed. The effect was dependent on the expression level of mutant $\Delta 18$, VLP release being completely inhibited at the higher level. Surprizingly, no effect was observed in the case of mutant $\Delta 1$, not even after transfection with the higher amount (Fig. 4).

\section{DISCUSSION}

Coronaviruses have the capacity to assemble uniform envelopes from their membrane proteins (Vennema et al. 1996). In this study we have exploited this feature to start an analysis of the primary structure requirements of the $\mathrm{M}$ protein for envelope formation. Together with the $\mathrm{N}$ protein, $\mathrm{M}$ is the most abundant virion component. The protein is 
considered to be the key element in virion assembly. It is essential not only for the formation of the viral envelope but also for drawing the nucleocapsid into the nascent particle by its interactions with the $\mathrm{N}$ protein. Its presumed role in localizing the budding of coronaviruses to membranes of the early exocytic pathway became questionable when the protein expressed by itself appeared to be transported to the Golgi complex (Klumperman et al., 1994). This role might be played by the E protein, which seems to be retained in preGolgi membranes when expressed independently (M. Raamsman, H. Vennema and P. Rottier, unpublished observations).

Our preliminary observations presented here demonstrate that assembly of virus like particles is critically sensitive to changes in either of the terminal domains of the $\mathrm{M}$ protein. Mutations not only in the carboxy-terminal tail but also in its amino-terminal domain can interfere with particle formation. The carboxy-terminal part of $M$ protrudes into the cytoplasm of cells. In infected cells it supposedly interacts with the nucleocapsid and ends up in the interior of the budded virion. Any truncation of this domain led to severe inhibition of particle formation in the VLP assay. The mere removal of just one terminal residue abolished assembly almost completely while no particles were detectable when two or more residues of the tail were lacking. Similarly fatal was the deletion of an internal domain, located in the amphiphilic region of the $\mathrm{M}$ protein (mutant $\Delta \mathrm{C}$ ). These effects are not likely to be the result of incorrect membrane integration or folding of the mutant $M$ proteins or of an inability to be transported as judged from their normal O-glycosylation patterns and their transport to the Golgi complex and beyond (mutant $\Delta 18$; data not shown).

How then do these mutations exert their effects? When considering the different interactions which the carboxy-terminal tail might entertain, a number of possibilites come up. The terminal residues might be involved in interactions between $\mathrm{M}$ molecules, as well as with $\mathrm{E}$, with membrane lipids or with host proteins somehow involved in the assembly process. Mutations, even the deletion of just one residue, could frustrate such interactions either directly or, when the mutation affects intramolecular interactions and, consequently, the proper formation of a necessary secondary structure in the M protein, indirectly. None of the various possibilities can as yet be excluded except probably the involvement of the carboxy-terminus in homotypic M-M interactions. Such interactions have been demonstrated earlier by Krijnse Locker et al. (1996) by showing the appearance in cells of large homomeric complexes of the expressed $\mathrm{M}$ protein, using sucrose density gradient analysis. In that study we found that such complexes, which presumably result from lateral interactions between $\mathrm{M}$ molecules, were also formed by a mutant $\mathrm{M}$ protein lacking its 22 carboxy-terminal residues.

The amino-terminus of $\mathrm{M}$ faces the lumen after the polypeptide has assembled in the ER membrane; in virions this domain is exposed on the outside (Rottier et al., 1984). Deleting the middle part of this domain (mutant $\Delta \mathrm{N}$ ) rendered the $\mathrm{M}$ protein unproductive in VLP formation. More subtle changes within this middle part gave divergent results. Replacement of the two conspicuous proline residues by alanines was without effect. In contrast, mutant $\mathrm{G}_{11} \mathrm{~N}_{13}$, meant to introduce a.o. an $\mathrm{N}$-glycosylation consensus sequence (which, by the way, appeared not to be functional), was strongly crippled in VLP assembly. Neither the deletion in mutant $\Delta \mathrm{N}$ nor the substitutions in mutant $\mathrm{G}_{11} \mathrm{~N}_{13}$ had affected the protein's fitness as judged from its normal exit from the ER and transport to the Golgi complex (immunofluorescence data mutant $\Delta \mathrm{N}$ : Rottier et al., 1990; normal O-glycosylation mutant $\mathrm{G}_{11} \mathrm{~N}_{13}$, Fig.3). This suggests that the effects of these mutations are not caused by drastic changes in the secondary or tertiary structure of the $\mathrm{M}$ molecule. An alternative possibility is that the amino-terminal domain is involved in intermolecular interactions, 
most likely between $\mathrm{M}$ molecules because little if anything of the membrane-integrated $\mathrm{E}$ protein is available on the luminal side (M.Raamsman, H.Vennema and P.Rottier, unpublished observations). For the human coronavirus (HCV-229E) M protein such interactions have indeed been demonstrated by Arpin and Talbot (1990) who showed the occurrence of covalently linked homodimers apparently formed by the single cysteine present in that molecule's ectodomain.

Two longstanding questions in the field are what the function is of the glycosylation of the $\mathrm{M}$ protein and why the protein in some coronaviruses carries $\mathrm{N}$-linked sugars as opposed to O-linked oligosaccharides in other viruses. Here we show that glycosylation per $s e$ is not required for the formation of the coronaviral envelope. Substitutions of the threonines at positions 4 and 5 completely abolished the O-glycosylation of the MHV M protein but did not affect VLP formation. This result eliminates one possible answer; the old questions, however, still remain.

An interesting observation made in our studies was that a mutant $\mathrm{M}$ protein that was itselve negative in VLP formation could interfere with the assembly of viral envelopes by wild-type $M$ protein. The mutant $\Delta 18$ appeared to poison the assembly process in a concentration-dependent way. Similar results were obtained with other cytoplasmic tail mutants but the effects became gradually weaker as the truncations were shorter (not shown), mutant $\Delta 1$ showing no detectable interference (Fig.3). These findings confirm our earlier conclusion that lateral interactions between $\mathrm{M}$ molecules are an important driving force in coronavirus particle formation (Krijnse Locker et al., 1995). They are also consistent with the finding that the carboxy-terminal tail is not required to form and stabilize these oligomeric interactions ( $i b i d$.). Oligomerization of $\mathrm{M}$ proteins is, however, obviously not sufficient for VLP formation; additional interactions involving the cytoplasmic domain are apparently required. The possible nature of these interactions was discussed above. Our observations are reminiscent of findings reported for Semliki Forest virus where p62E1 heterodimers, nucleocapsid binding-deficient due to mutations in the cytoplasmic domain of the p62 protein, were found to inhibit normal virus budding also in a concentration dependent manner (Ekström et al., 1994).

It is clear from this study that the VLP system has great potential for the analysis of the structural requirements of the individual coronaviral membrane proteins as well as for the investigation of their interactions during coronavirus envelope assembly. Whether, and to what extent, observations made in this system can be extrapolated to the assembly of coronavirions is still unclear. This will require the introduction of selected mutations into the coronaviral genome, a major challenge for the future.

\section{ACKNOWLEDGMENTS}

We are gratefull to Peggy Roestenberg and Marèl de Wit for their assistence in part of the experimental work. The investigations were supported by the Netherlands Foundation for Chemical Research (SON) with the financial aid from the Netherlands Organization for Scientific Research (NWO).

\section{REFERENCES}

Arpin, N., and Talbot, P.J., 1990, Molecular characterization of the 229E strain of human coronavirus, Adv. Exp. Med. Biol. 276:73-80. 
de Vries, A.A.F., Raamsman, M.J.B., van Dijk, H.A., Horzinek, M.C., and Rottier, P.J.M., 1995, The small envelope glycoprotein $(\mathrm{Gs})$ of equine arteritis virus folds into three distinct monomers and a disulfide-linked dimer, J. Virol. 69:344-3448.

Delchambre, M., Gheysen, D., Thines, D., Thiriart, C., Jacobs, E., Verdin, E., Horth, M., Burny, A., and Bex, F., 1989, The Gag precursor of simian immunodeficiency virus assembles into virus-like particles, EMBO J. 8:2653-2660.

Ekström, M., Liljeström, P., and Garoff, H., 1994, Membrane protein lateral interactions control Semliki Forest virus budding, EMBO J. 13:1058-1064.

Gheysen, D., Jacobs, E., de Foresta, A., Thiriart, C., Francotte, M., Thines, D., and De Wilde, M., 1989, Assembly and release of HIV-1 precursor Pr55gag virus-like particles from recombinant baculovirus-infected cells, Cell 59:103-112.

Hobman, T.C., Lundstrom, M.L., Mauracher, C.A., Woodward, L., Gilliam, S., and Farquhar, M.G., 1994, Assembly of rubella virus structural proteins into virus-like particles in transfected cells, Virology 115:574-585.

Klumperman, J., Krijnse Locker, J., Meijer, A., Horzinek, M.C., Geuze, H.J., and Rottier, P.J.M., 1994, Coronavirus $M$ proteins accumulate in the Golgi complex beyond the site of virion buddin, J. Virol. 68:6523-6534.

Krijnse Locker, J., Opstelten, D.-J.E., Ericsson, M., Horzinek, M.C., and Rottier, P.J.M., 1995, Oligomerization of a trans-Golgi/trans-Golgi network retained protein occurs in the Golgi complex and may be part of its retention, J. Biol. Chem. 270:88158821.

Mead, D.A., Szczesna-Skorupa, E., and Kemper, B., 1986, Single-stranded DNA 'blue' T7 promotor plasmids: A versatile tandem promotor system for cloning and protein engineering, Prot. Engineering 1:67-74.

Mebatsion, T., König, M., and Conzelman, K.-K., 1996, Budding of rabies virus particles in the absence of the spike glycoprotein, Cell 84:941-951.

Rottier, P.J.M., Horzinek, M.C., and van der Zeijst, B.A.M., 1981, Viral protein synthesis in mouse hepatitis virus strain A59-infected cells: effect of tunicamycin, J. Virol. 40:350-357.

Rottier, P.J.M., Brandenburg, D., Armstrong, J., van der Zeijst B.A.M., and Warren, G., 1984, Assembly in vitro of a spanning membrane protein of the endoplasmatic reticulum: the E1 glycoprotein of coronavirus mouse hepatitis virus A59, Proc. Natl. Acad. Sci. USA 81:1421-1425.

Rottier, P.J.M., Krijnse Locker, J., Horzinek, M.C., and Spaan, W.J.M., 1990. Expression of MHV-A59 M glycoprotein: Effects of deletions on membrane integration and intracellular transport, Adv. Exp. Med. Biol. 276:127-135.

Rottier, P.J.M., 1995, The coronavirus membrane protein, p. 115-139. In S. G. Siddell (ed.), The Coronaviridae, Plenum Press, New York.

Schalich, J., Allison, S.L., Stiasny, K., Mandl, C.J., Kunz, C., and Heinz, F.X., 1996, Recombinant subviral particles from tick-borne encephalitis virus are fusogenic and provide a model system for studying flavivirus envelope glycoprotein functions, J. Virol. 70:4549-4557.

Simon, K., Lingappa, V.R., and Ganem, D., 1988, Secreted hepatitis B surface antigen polypeptides are derived from a transmembrane precursor, J. Cell Biol. 107:2163-2168.

Suomalainen, M., Liljeström, P., and Garoff, H., 1992, Spike protein-nucleocapsid interations drive the budding of alphaviruses, J. Virol. 66:4737-4747.

Vennema, H., Godeke, G.-J., Rossen, J.W.A., Voorhout, W.F., Horzinek, M.C., Opstelten, D.-J.E., and Rottier, P.J.M., 1996, Nucleocapsid-independent assembly of corona virus-like particles by co-expression of viral envelope protein genes, EMBO J. 15:2020-2028. 